Canadian

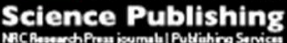

Biochemistry and Cell Biology Biochimie et biologie cellulaire

\title{
Method Comparison for Analyzing Wound Healing Rates
}

\begin{tabular}{|r|l|}
\hline Journal: & Biochemistry and Cell Biology \\
\hline Manuscript ID & bcb-2016-0163.R3 \\
\hline Danuscript Type: & Note \\
\hline Complete List of Authors: & $\begin{array}{l}\text { Dhillon, Prabhpreet; Simon Fraser University, Molecular Biology and } \\
\text { Biochemistry } \\
\text { Li, Xinyin; Simon Fraser University, Chemistry } \\
\text { Sanes, Jurgen; Simon Fraser University, Chemistry } \\
\text { Akintola, Oluwafemi; Simon Fraser University, Chemistry } \\
\text { Sun, Bingyun; Simon Fraser University, Chemistry and Molecular and } \\
\text { Cellular Biology }\end{array}$ \\
\hline Keyword: & $\begin{array}{l}\text { wound healing scratch assay, cell migration rate, Chinese Hamster Ovary } \\
\text { cells, N-acetylglucosamine (GlcNAc) }\end{array}$ \\
\hline &
\end{tabular}

SCHOLARONE ${ }^{\text {M }}$

Manuscripts 


\section{Method Comparison for Analyzing Wound Healing Rates}

2 Prabhpreet K. Dhillon ${ }^{a}$, Xinyin Li $^{b}$, Jurgen T. Sanes ${ }^{b}$, Oluwafemi S. Akintola ${ }^{b}$, Bingyun Sun ${ }^{a, b, c *}$

$4 \quad{ }^{a}$ Department of Molecular Biology and Biochemistry, Simon Fraser University, Burnaby, BC, Canada

$5 \quad{ }^{\mathrm{b}}$ Department of Chemistry, Simon Fraser University, Burnaby, BC, Canada

$6 \quad{ }^{\mathrm{c}}$ Centre for Cell Biology, Development, and Disease, Simon Fraser University, BC, Canada

* Correspondence: Bingyun Sun, Simon Fraser University, Burnaby, BC, Canada, Tel.: (778) 782-9097,

$9 \quad$ Fax: (778) 782-3765, Email: bingyun_sun@sfu.ca

10

11

12

13

14

15

16

17 

ABSTRACT Wound healing scratch assay is a frequently used method to characterize cell migration, which is an important biological process in the course of development, tissue repair, and immune response for

21 example. The measurement of wound healing rate, however, varies among different studies. Here we

22 summarized these measurements into three types: I) Direct Rate Average; II) Regression Rate Average;

23 and III) Average Distance Regression Rate. Using Chinese Hamster Ovary (CHO) cells as a model, we 24 compared the three types of analyses on quantifying the wound closing rate, and discovered that type I $25 \&$ III measurements are more resistant to outliers, and type II analysis is more sensitive to outliers. We 26 hope this study can help researchers to better use this simple yet effective assay.

27

28 KEYWORDS

29 wound healing scratch assay, cell migration rate, Chinese Hamster Ovary cells, N-acetylglucosamine 30 (GlcNAc) 
34

35

36

37

38

39

40

41

42

43

\section{INTRODUCTION}

Cell migration is fundamental to normal development and homeostasis of tissues and organs as well as to various pathological states, such as cancer metastasis. In developing embryo, cell migration plays an essential role in gastrulation and organogenesis; in developed organs, migration is also crucial to tissue regeneration (Lauffenburger, D. A. et al. 1996). The process itself is a complex phenomenon that requires synchronization and coordination of various cellular events, such as cell polarization, protrusion, adhesion, translocation of the cell body and the retraction of its trailing edge (Lauffenburger, D. A. et al. 1996; Horwitz, R. et al. 2003). Due to the paramount physiological importance, understanding the underlying biology of cell migration is of interest to scientists for a number of therapeutic applications.

Several in vitro assays have been developed to characterize this process. Transwell migration assay (Boyden chamber assay), cell exclusion zone assay, fence assay, micro-carrier bead assay and wound healing assay are a few examples (Kramer, N. et al. 2013). Among these, wound healing scratch assay is one of the simplest, economical and well-studied methods to measure cell migration in vitro that to some extent mimics in vivo wound healing (Lampugnani, M. G. 1999; Liang, C.-C. et al. 2007). It operates on the idea that formation of an artificial gap or scratch in a confluent monolayer of cells will result in the migration of cells on the wound edge towards the center of the gap until the scratch is closed (Zahm, J.-

M. et al. 1997). This response is due to the disruption of cell-cell contacts resulting in an increased concentration of growth factors at the wound edge (Wong, M. K. et al. 1988; Coomber, B. L. et al. 1990; Zahm, J.-M. et al. 1997). The healing then takes place through a combination of migration and proliferation until the cell-cell contacts are reestablished (Wong, M. K. et al. 1988; Yarrow, J. C. et al. 2004). 
56 Due to its ease of operation, it has been widely adapted for studying wound healing. The method of

57 computing the rate, however, has varied from study to study. The scratching of a confluent cell surface

58 is likely to create uneven boundary with the intrinsic variation. The moving front of cells is therefore not

59 even, and multiple measurements are necessary at different locations over time to assess the average rate

60 (Maini, P. K. et al. 2004). Studies on the migration of single cells have also been carried out to more

61 accurately examine the rate (Friedl, P. et al. 2003; Ridley, A. J. et al. 2003; Friedl, P. et al. 2010). In

62 these studies, the sequence varies on computing the gap closing rate through regression analysis and on

63 averaging the obtained rates. Recently, single-time-point measurement also emerged for quick

64

65

66

67

68

69

70

71

72

73

74

75

76 assessment of migration difference among studied conditions, in which regression analysis as a function of time on wound closing is omitted (Dowling, Catríona M. et al. 2014; Shafqat-Abbasi, H. et al. 2016).

We for the first time attempted to catalog these measurements of wound closing rate into three types: I)

Direct Rate Average; II) Regression Rate Average and III) Average Distance Regression Rate. Using

Chinese Hamster Ovary (CHO) cells as a model, and using regular and starving media as two conditions, we compared the difference of wound closing rate measured by all three methods in varied

$\mathrm{N}$-acetylglucosamine (GlcNAc) concentrations. We demonstrated that the rates computed by all

methods are similar with slight difference when no outliers existed. Interestingly, we also discovered that with outliers, type II analysis was affected more, therefore, more sensitive to inconsistent measurements; whereas the other two analyses were more robust to outliers.

\section{MATERIALS AND METHODS}

Cell culture 
77 CHO-K1 cells were cultured in regular DMEM media (Lonza 12614F12) supplemented with 10\% Fetal

78 Bovine Serum (FBS), 1\% L-glutamine and 1\% antibiotics (streptomycin and penicillin) obtained from

79 Invitrogen. Prior to scratching, cells were incubated at $37^{\circ} \mathrm{C}$ and $5 \% \mathrm{CO}_{2}$ to reach $\sim 90 \%$ confluency.

80 Condition 1

81 Cells were cultured with treatment DMEM media (Sigma, D5030-10L) supplemented with pyruvate, L-

82 glutamine, $\mathrm{NaHCO}_{3}, 10 \% \mathrm{FBS}, 4.5 \mathrm{~g} / \mathrm{L}$ glucose and various concentrations of $\mathrm{N}$-acetylglucosamine

83 (GlcNAc) obtained from Sigma.

84 Condition 2

85 Cells were incubated with starving media, which was treatment DMEM media without FBS and lowered 86 glucose at $1 \mathrm{~g} / \mathrm{L}$ for 24 hours prior to scratching. After scratching, the cells were switched to $10 \%$ FBS

87 supplemented treatment media with still lowered glucose at $1 \mathrm{~g} / \mathrm{L}$.

Wound healing scratch assay

89 Two vertical wounds were created on the cell monolayer using P100 pipette tip (Liang, C.-C. et al.

90 2007) in every culture plate. Wound closing was monitored over defined time intervals by digital camera

91 (Nikon Coolpix S6200). The recorded wound length was analyzed by imageJ (http://imagej.nih.gov/ij/).

92 Four spots were chosen in each plate to compute the healing rate in condition 1; and 3 spots were used in 93 condition 2.

94 Analysis of wound healing rate

\section{Type I analysis (Direct Rate Average):}

96 The wound healing rate $(\mathrm{R})$ at any spot in one plate with defined time was computed by the healing

97 distance $\left(\Delta \mathrm{W}_{\mathrm{t}}\right)$ over time $(\mathrm{t})$, i.e. $\mathrm{R}=\Delta \mathrm{W}_{\mathrm{t}} / \Delta \mathrm{t}$ 
98 The average wound healing rate, $\overline{\mathrm{R}}_{\text {plate}}$, over different spots at defined time, was computed as following:

$$
\overline{\mathrm{R}}_{\text {plate }}=\frac{\sum \mathrm{R}}{\mathrm{N}_{\text {spots }}}
$$

100

101

102

103

104

105

106

107

108

109

Where $\mathrm{N}_{\mathrm{spots}}$ is the number of spots chosen for monitoring over time. The overall average wound healing rate of all detected time points was calculated by the equation below:

$$
\overline{\mathrm{R}}_{\text {total }}=\frac{\sum \overline{\mathrm{R}}_{\text {plate }}}{\mathrm{N}_{\text {time points }}}
$$

Where $\overline{\mathrm{R}}_{\text {total }}$ is the final wound healing rate, and $\mathrm{N}_{\text {time points }}$ is the number of time points chosen in the experiment to monitor the wound closure. For single time-point analysis, the value of $\mathrm{N}_{\text {time points }}$ is one.

\section{Type II analysis (Regression Rate Average):}

We plotted the wound distance (W) at a given spot as a function of time and used linear regression to obtain the slope, which is the wound healing rate per spot $\left(\overline{\mathrm{R}}_{\mathrm{spot}}\right)$. Then, the plate wound healing rate was computed by averaging the spot wound healing rate at all monitored spots using equation below:

$$
\overline{\mathrm{R}}_{\text {total }}=\frac{\sum \overline{\mathrm{R}} \mathrm{spot}}{\mathrm{N}_{\text {spots }}}
$$

\section{Type III analysis (Average Distance Regression Rate):}

We first averaged the wound length $(\bar{W})$ at each time point for all spots of a plate. Then, we calculated the closed average wound $\left(\Delta \overline{W_{t}}\right)$ at each time point of all spots of a plate:

$$
\Delta \overline{\mathrm{W}}_{\mathrm{t}}=\overline{\mathrm{W}}_{0}-\overline{\mathrm{W}}_{\mathrm{t}}
$$

Where $\bar{W}_{0}$ is the original wound length at 0 hour, and $\bar{W}_{t}$ is the wound length at $\mathrm{t}$ hour. Finally, we plotted the $\Delta \overline{\mathrm{W}}$ as a function of $\mathrm{t}$, and used linear regression to obtain the total wound healing rate $\mathrm{R}_{\text {total }}$. 


\section{Significance analysis:}

118 Microsoft Graphpad Prism software was used for significance analysis. One-way ANOVA Tukey's test 119 was used to analyze rate changes across multiple GlcNAc concentrations within the same treatment 120 condition. Unpaired and two-tail t test was used to analyze significant changes between two conditions.

\section{RESULTS AND DISCUSSION}

Comparison of methods used for analysis

125 To compare different rate analysis methods, we used CHO cells, a common mammalian cell line that is 126 frequently used as a host for expression of specific proteins (Zhu, J. 2012). Therefore, CHO cells have 127 been used to investigate the function of exogenous proteins in migration (Hori, A. et al. 2001; Ganguly, A. et al. 2012). Under two slightly different conditions, we monitored the wound healing as shown in

129 Figs. 1 \& 2, respectively. We used three types of analyses to quantify the wound healing rate and compared their results. The three methods differ in how and when the migration rate is computed. Type I analysis directly computes the migration rate at given time point by dividing the wound length over

132 time. Then wound closing rates at different spots and different time points can be averaged for overall 133 healing rate of the population. This analysis was frequently applied recently when single time point was 134 selected for assessing migration rate (Dowling, Catríona M. et al. 2014; Shafqat-Abbasi, H. et al. 2016). 135 Type II analysis uses linear regression to obtain healing rate over several time points for every spot.

136 Then the rate of different spots is averaged for final closing rate of the population, which has been 137 employed to study single-cell migration (Komuro, H. et al. 1995). Type III analysis averages the wound 138 length across multiple spots first, and then uses the average distance over time for regression analysis to 
139 obtain the overall healing rate (Stokes, C. L. et al. 1991). Type III analysis is the common method used 140 in scratching assay when multiple time points are used for analysis.

141 To further examine the changes of wound healing rate, we treated CHO cells with various

142 concentrations of GlcNAc. GlcNAc is well known for its structural role on the cell surface (Naseem, S.

143 et al. 2012). It is a key component of extracellular matrix of mammalian cells, and participates in wound 144 healing events in metazoan (Janik, M. E. et al. 2010; Hart, G. W. et al. 2011; Konopka, J. B. 2012). The

145 obtained wound healing rates under various GlcNAc concentrations in condition 1 are summarized in 146 Fig. 1. In the figure, except for Fig. 1B, 10 and $50 \mathrm{mM}$ GlcNAc concentrations, all three types of

147 analyses showed relatively good agreement to each other. Our measured closing rates of CHO cells 148 without GlcNAc by all three analysis methods were between $0.01-0.03 \mathrm{~mm} / \mathrm{hour}$, which was in the same 149 range as previous reports (Dübe, B. et al. 2001) suggesting our assay was reliable.

150 To note in the experiments of Fig 1B, two of the four spots under 10mM and 50mM GlcNAc showed 151 early closure of the wound. The cause of early closure was likely due to the insufficient removal of 152 extracellular matrix structure. It is known that the inconsistent scratching can cause the variation of the 153 subsequent cell migration if the cell basal membrane or extracellular matrix were not removed 154 effectively (Liang, C.-C. et al. 2007). The absence of early closure in the results of Figs. 1C \& 1D 155 supported that the two incidences observed in Fig. 1B were outliers.

156 Interestingly in Fig. 1B, results from analysis I \& III were similar to each other across different GlcNAc 157 concentrations regardless of the outliers, whereas only those from analysis II showed large difference at 158 GlcNAc concentrations with abnormal migration (outliers). These results suggested that analysis II is 159 more prone to outlier effects than the other two types of analyses. 
161 In addition to condition 1, we also designed starvation condition 2 to monitor CHO cell wound closing

162 rate under varied GlcNAc concentrations as shown in Fig. 2. The reason was because extracellular

163 GlcNAc was known to slowdown cell migration and proliferation (Runyan, R. B. et al. 1986; Atnip, K.

164 D. et al. 1987; Viola, S. et al. 2008). Our results under condition 1 in Fig. 1 only showed a small drop of 165 closing rate at higher GlcNAc concentration, and ANOVA analysis of results from three sets of repeated 166 experiments (Fig. 1B) across varied GlcNAc concentration did not show significant difference 167 regardless of analysis methods. We wanted to verify whether our analysis can identify any statistically 168 significant rate difference. Towards this end, we designed condition 2, i.e. the starving condition. 169 Condition 2 was different from condition 1 in two ways: first, cells in condition 2 were starved for 24 170 hours before being cultured in treatment media containing different concentrations of GlcNAc; and 171 second, glucose concentration in the conditional 2 media was $1 \mathrm{~g} / \mathrm{L}$ as compared to $4.5 \mathrm{~g} / \mathrm{L}$ in condition 172 1. The example images and the computed migration rates by three different methods are shown in Fig. 2.

173 Interestingly, type II analysis again identified an outlier in Fig. 2B under 2mM GlcNAc treatment 174 further validated its sensitivity. We compared the average migration rate in two biological replicates 175 between conditions $1 \& 2$ (excluding the results with outliers) obtained by sensitive type II analysis for 176 both the control and 10mM-GlcNAc treatment as shown in Fig. 3. A significant reduction of wound 177 healing rate $(\mathrm{P}<0.01, \mathrm{t}$ test $)$ was observed in condition 2 compared to condition 1 under the same 178 GlcNAc concentration. For GlcNAc effect, no statistical difference was observed between 0 and $10 \mathrm{mM}$ 179 GlcNAc in condition 1, but a decrease of rate in GlcNAc was observed in condition 2 (P: 0.01 0.02, t 180 test). A result suggested that our analysis was able to assess the rate change.

181 The decreased wound closing rate of cells under starving condition was congruent with the existing 182 knowledge. Starvation is known to arrest cells at G0/G1 phase of the cell cycle (Pardee, A. B. 1974; 183 Khammanit, R. et al. 2008; Rosner, M. et al. 2011), and the hypoglycemia introduced by the reduced 
184 glucose concentration in culture media can impair normal wound healing (Hayashi, J. N. et al. 1991;

185 McDermott, A. M., Kern, T. S., \& Murphy, C. J. 1998; Liu, Y. et al. 2003). The reduction of wound

186 healing rate in condition 2 allowed us to disclose the reported GlcNAc effect (Runyan, R. B. et al. 1986;

187 Atnip, K. D. et al. 1987; Viola, S. et al. 2008) that were otherwise confounded by the large variance in 188 the experiments in condition 1.

\section{CONCLUSION}

191 We cataloged three different methods of quantifying the wound closing rate in scratching assay and 192 discovered their differential capacity to tolerate outliers. We hope this study can help other researchers 193 to better analyze the results generated from this simple yet effective assay.

\section{ACKNOWLEDGEMENTS}

195 This work was financially supported by Simon Fraser University work-study program and Canadian 196 Stem Cell Network. 
201

202

203

204

205

206

207

208

209

210

211

212

213

214

215

216

217

218

\section{REFERENCES}

Atnip, K. D., Mahan, J. T. and Donaldson, D. J. 1987. Role of carbohydrates in cell-substrate interactions during newt epidermal cell migration. Journal of Experimental Zoology. 243(3): 461-471.

Coomber, B. L. and Gotlieb, A. I. 1990. In vitro endothelial wound repair. Interaction of cell migration and proliferation. Arteriosclerosis, Thrombosis, and Vascular Biology. 10(2): 215-222.

Dowling, Catríona M., Herranz Ors, C. and Kiely, Patrick A. 2014. Using real-time impedance-based assays to monitor the effects of fibroblast-derived media on the adhesion, proliferation, migration and invasion of colon cancer cells. Bioscience Reports. 34(4): e00126.

Dübe, B., Lüke, H. J., Aumailley, M. and Prehm, P. 2001. Hyaluronan reduces migration and proliferation in $\mathrm{CHO}$ cells. Biochimica et Biophysica Acta (BBA) - Molecular Cell Research. 1538(2-3): 283-289.

Friedl, P. and Wolf, K. 2003. Tumour-cell invasion and migration: diversity and escape mechanisms. Nat Rev Cancer. 3(5): 362-374.

Friedl, P. and Wolf, K. 2010. Plasticity of cell migration: a multiscale tuning model. The Journal of Cell Biology. 188(1): 11-19.

Ganguly, A., Yang, H., Sharma, R., Patel, K. D. and Cabral, F. 2012. The role of microtubules and their dynamics in cell migration. J Biol Chem. 287(52): 43359-43369. 
219

220

221

222

223

224

225

226

227

228

229

230

231

232

233

234

235

236

237

238

Hart, G. W., Slawson, C., Ramirez-Correa, G. and Lagerlof, O. 2011. Cross Talk Between OGlcNAcylation and Phosphorylation: Roles in Signaling, Transcription, and Chronic Disease. Annual Review of Biochemistry. 80: 825-858.

Hayashi, J. N., Ito, H., Kanayasu, T., Asuwa, N., Morita, I., Ishii, T. and Murota, S.-i. 1991. Effects of glucose on migration, proliferation and tube formation by vascular endothelial cells. Virchows Archiv B. 60(1): 245-252.

Hori, A., Honda, S., Asada, M., Ohtaki, T., Oda, K., Watanabe, T., Shintani, Y., Yamada, T., Suenaga, M., Kitada, C., Onda, H., Kurokawa, T., Nishimura, O. and Fujino, M. 2001. Metastin suppresses the motility and growth of CHO cells transfected with its receptor. Biochem Biophys Res Commun. 286(5): 958-963.

Horwitz, R. and Webb, D. 2003. Cell migration. Current Biology. 13(19): R756-R759.

Janik, M. E., Lityńska, A. and Vereecken, P. 2010. Cell migration-The role of integrin glycosylation. Biochimica et Biophysica Acta (BBA) - General Subjects. 1800(6): 545-555.

Khammanit, R., Chantakru, S., Kitiyanant, Y. and Saikhun, J. 2008. Effect of serum starvation and chemical inhibitors on cell cycle synchronization of canine dermal fibroblasts. Theriogenology. 70(1): $27-34$.

Konopka, J. B. 2012. N-Acetylglucosamine Functions in Cell Signaling. Scientifica. 2012: 489208.

Kramer, N., Walzl, A., Unger, C., Rosner, M., Krupitza, G., Hengstschläger, M. and Dolznig, H. 2013. In vitro cell migration and invasion assays. Mutation Research/Reviews in Mutation Research. 752(1): 10-24. 
239 Lampugnani, M. G. (1999). Cell Migration into a Wounded Area In Vitro. Adhesion Protein Protocols.

240 E. Dejana and M. Corada. Totowa, NJ, Humana Press: 177-182.

241 Lauffenburger, D. A. and Horwitz, A. F. 1996. Cell Migration: A Physically Integrated Molecular $242 \quad$ Process. Cell. 84(3): 359-369.

243 Liang, C.-C., Park, A. Y. and Guan, J.-L. 2007. In vitro scratch assay: a convenient and inexpensive 244 method for analysis of cell migration in vitro. Nat. Protocols. 2(2): 329-333.

245 Liu, Y., Song, X.-D., Liu, W., Zhang, T.-Y. and Zuo, J. 2003. Glucose deprivation induces mitochondrial dysfunction and oxidative stress in PC12 cell line. Journal of Cellular and Molecular Medicine. 7(1): 49-56.

Maini, P. K., McElwain, D. L. and Leavesley, D. I. 2004. Traveling wave model to interpret a woundhealing cell migration assay for human peritoneal mesothelial cells. Tissue Eng. 10(3-4): 475482.

McDermott, A. M., Kern, T. S., \& Murphy, C. J. 1998. Effect of elevated extracellular glucose on adhesion and migration of SV40 transformed human corneal epithelial cells. Current Eye Research. 9: 924-932.

Naseem, S., Parrino, S. M., Buenten, D. M. and Konopka, J. B. 2012. Novel roles for GlcNAc in cell signaling. Communicative \& Integrative Biology. 5(2): 156-159. the National Academy of Sciences. 71(4): 1286-1290. 
Rakic, H. K. a. P. 1995. Dynamics of Granule Cell Migration: A Confocal Microscopic Study in Acute Cerebellar Slice Preparations The Journal of Neuroscience. 15(2): 1110-1120.

Ridley, A. J., Schwartz, M. A., Burridge, K., Firtel, R. A., Ginsberg, M. H., Borisy, G., Parsons, J. T. and Horwitz, A. R. 2003. Cell Migration: Integrating Signals from Front to Back. Science. 302(5651): 1704-1709.

Rosner, M. and Hengstschläger, M. 2011. Nucleocytoplasmic localization of p70 S6K1, but not of its isoforms $\mathrm{p} 85$ and $\mathrm{p} 31$, is regulated by TSC2/mTOR. Oncogene. 30(44): 4509-4522.

Runyan, R. B., Maxwell, G. D. and Shur, B. D. 1986. Evidence for a novel enzymatic mechanism of neural crest cell migration on extracellular glycoconjugate matrices. The Journal of Cell Biology. 102(2): 432-441.

Shafqat-Abbasi, H., Kowalewski, J. M., Kiss, A., Gong, X., Hernandez-Varas, P., Berge, U., JafariMamaghani, M., Lock, J. G. and Strömblad, S. 2016. An analysis toolbox to explore mesenchymal migration heterogeneity reveals adaptive switching between distinct modes. eLife. 5: e11384.

Stokes, C. L., Lauffenburger, D. A. and Williams, S. K. 1991. Migration of individual microvessel endothelial cells: stochastic model and parameter measurement. J Cell Sci. 99(Pt 2): 419-430.

Viola, S., Consoli, G. M. L., Merlo, S., Drago, F., Sortino, M. A. and Geraci, C. 2008. Inhibition of rat glioma cell migration and proliferation by a calix[8]arene scaffold exposing multiple GlcNAc and ureido functionalities. Journal of Neurochemistry. 107(4): 1047-1055. 
277 Wong, M. K. and Gotlieb, A. I. 1988. The reorganization of microfilaments, centrosomes, and 278 microtubules during in vitro small wound reendothelialization. The Journal of Cell Biology.

279

Yarrow, J. C., Perlman, Z. E., Westwood, N. J. and Mitchison, T. J. 2004. A high-throughput cell migration assay using scratch wound healing, a comparison of image-based readout methods. BMC Biotechnology. 4(1): 1-9. migration and proliferation during the in vitro wound repair of the respiratory epithelium. Cell Motility and the Cytoskeleton. 37(1): 33-43. advances. 30(5): 1158-1170. 


\section{FIGURE CAPTIONS}

292 Fig. 1. A. Images of scratch wound healing over time under different GlcNAc concentrations in

293 condition 1 . The vertical lines on the images indicate the boundary of wound at 0 hour. Healing distance

294 was measured relative to this boundary. B-D. Comparison of migration rate computed by three types of

295 analysis in three repeated assays under condition 1, and * labels the outliers. Number of spots used (N)

296 were 4, error bar is the standard deviation. In type III analysis, linear regression analysis $r^{2}$ values for

297 migration rates computed for GlcNAc concentration from 0 to $50 \mathrm{mM}$ are all above 0.8 .

298 Fig. 2. Images of scratch wound healing over time under different GlcNAc concentrations in condition

299 2. The vertical lines on the images indicate the boundary of wound at 0 hour. Healing distance was

300 measured relative to this boundary. B-D. Comparison of migration rate computed by three types of

301 analysis in three repeated assays under condition 2, and * labels the outlier. Number of spots used (N)

302 were 3, error bar is the standard deviation. In type III analysis, linear regression analysis $\mathrm{r}^{2}$ values for

303 migration rates computed for GlcNAc concentration from 0 to $10 \mathrm{mM}$ are all above 0.9 .

304 Fig. 3. Comparison of wound healing rates between condition 1 and 2 using analysis type II. Error bar is 305 the standard deviation of two biological replicates (C \& D in both Figs. 1 \& 2) without outliers. 

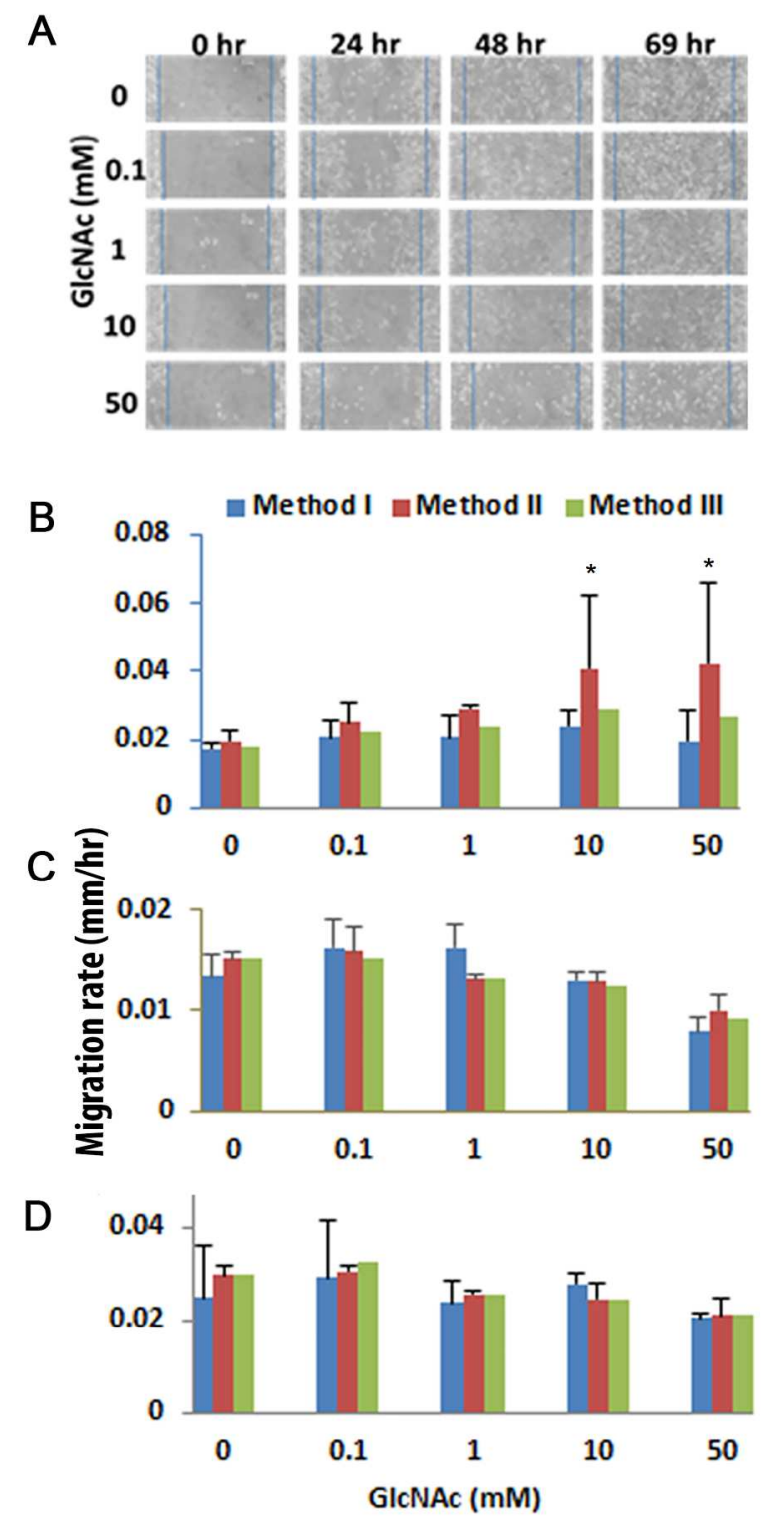

Fig. 1. A. Images of scratch wound healing over time under different GlcNAc concentrations in condition 1. The vertical lines on the images indicate the boundary of wound at 0 hour. Healing distance was measured relative to this boundary. B-D. Comparison of migration rate computed by three types of analysis in three repeated assays under condition 1 , and * labels the outliers. Number of spots used (N) were 4, error bar is the standard deviation. In type III analysis, linear regression analysis $r 2$ values for migration rates computed for GlcNAc concentration from 0 to $50 \mathrm{mM}$ are all above 0.8 .

$$
161 \times 322 \mathrm{~mm}(300 \times 300 \mathrm{DPI})
$$


A
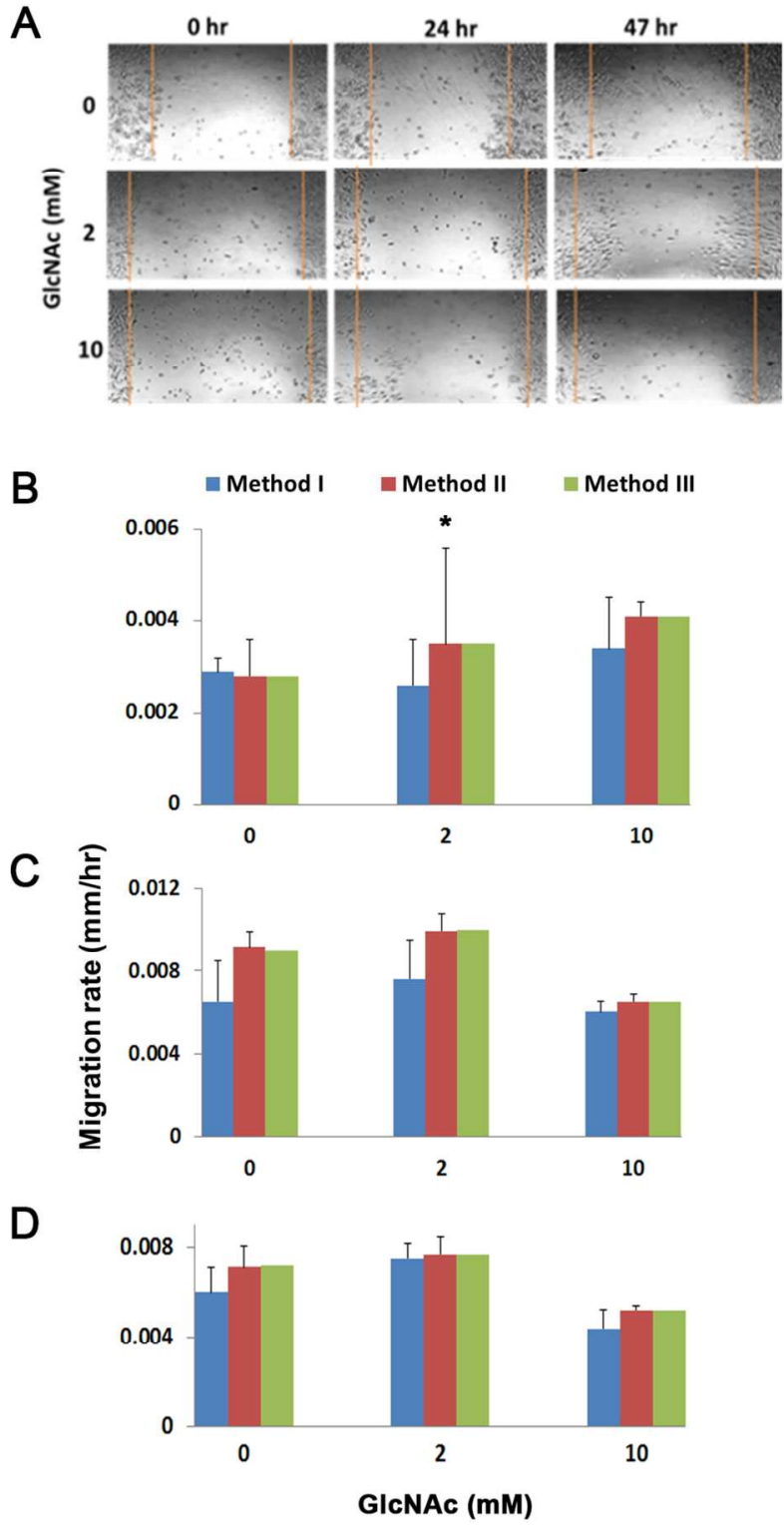

Fig. 2. Images of scratch wound healing over time under different GlcNAc concentrations in condition 2. The vertical lines on the images indicate the boundary of wound at 0 hour. Healing distance was measured relative to this boundary. B-D. Comparison of migration rate computed by three types of analysis in three repeated assays under condition 2, and * labels the outlier. Number of spots used (N) were 3, error bar is the standard deviation. In type III analysis, linear regression analysis $r 2$ values for migration rates computed for GlcNAc concentration from 0 to $10 \mathrm{mM}$ are all above 0.9 .

$102 \times 187 \mathrm{~mm}(300 \times 300$ DPI $)$ 


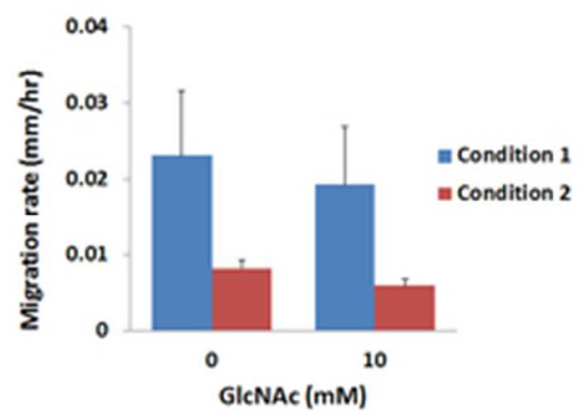

Fig. 3. Comparison of wound healing rates between condition 1 and 2 using analysis type II. Error bar is the standard deviation of two biological replicates (C \& D in both Figs. $1 \& 2$ ) without outliers.

$19 \times 13 \mathrm{~mm}(300 \times 300$ DPI $)$ 